\title{
A comparison study for solving systems of high-order ordinary differential equations with constants coefficients by exponential Legendre collocation method
}

\author{
Mohamed Elarbi Benattia ${ }^{a^{*}}$, Kacem Belghaba ${ }^{a}$ and Noureddine Bouteraa ${ }^{b}$ \\ ${ }^{\mathrm{a}}$ Laboratory of Mathematics and its Applications (LAMAP) University of Oran 1, Ahmed Ben Bella, Algeria \\ ${ }^{\mathrm{b}}$ Laboratory of Fundamental and Applied Mathematics of Oran (LMFAO) University of Oran 1, Ahmed Ben Bella, Algeria \\ ${ }^{*}$ Corresponding author E-mail: mohamed.benattia74@yahoo.com
}

\author{
Article Info \\ Keywords: Exponential Legendre func- \\ tions, System of ordinary differential \\ equations, Collocation method, System \\ of linear algebraic equations \\ 2010 AMS: 65D15, 65L05 \\ Received: 17 April 2018 \\ Accepted: 5 June 2018 \\ Available online: 30 June 2018
}

\begin{abstract}
In this article we are interested to study the use of the Legendre exponential (EL) collocation method to solve systems of high order linear ordinary differential equations with constant coefficients. The method transforms the system of differential equations and the conditions given by matrix equations with constant coefficients a new system of equations that corresponds to the system of linear algebraic equations which can be solved . Numerical problems are given to illustrate the validity and applicability of the method. For obtaining the approximate solution Maple software is used.
\end{abstract}

\section{Introduction}

Legendre polynomials are one of the most important special functions, which are widely used in numerical analysis[6]. The Legendrre polynomials are orthogonal with respect to the weight function 1 on the interval $[-1 ; 1]$ and the recurrence relations is

$$
L_{0}(x)=1, L_{1}(x)=x, L_{n+1}(x)=\frac{2 n+1}{n+1} x L_{n}(x)-\frac{n}{n+1} L_{n-1}(x), n \geqslant 1 .
$$

One of the applications of Legendre polynomials is the solution of ordinary differential equations with boundary conditions with collocation points. Under a transformation that maps the interval $[-1 ; 1]$ into a semi-infinite domain $[0 ; \infty)$, we applied spectral methods to solve problems on semi-infinite intervals $[3,1,8,9]$. In their studies, the basis functions called exponential Legendre (EL) functions $E_{n}(t)$ are orthogonal in $[-1 ; 1]$. The EL functions are defined as

$$
\begin{gathered}
E_{0}(t)=1, E_{1}(t)=1-2 e^{-t} \\
E_{n+1}(t)=\frac{2 n+1}{n+1}\left(1-2 e^{-t}\right) E_{n}(t)-\frac{n}{n+1} E_{n-1}(t), n \geqslant 1 .
\end{gathered}
$$

Recently, we reported a new operational matrix of derivatives of EL functions for solving ODEs in semi infinite domains. In this paper we applied the matrix of derivative mentioned in [3] to solve systems of ordinary differential equations defined on the whole range by means of collocation method. The organization of this paper is as follows. In Section 2, Preliminaries introduced while in Section 3 Properties of the exponential Legendre (EL) functions are presented. In Section 4, we formulated the fundamental matrix relation based on collocation Points. In Section 5, method of solution is presented. Section 6 contains numerical illustrations. 


\section{Preliminaries}

In this paper, we considered a system of $k$ linear differential equations with variable coefficients of the $m t h$ order in the form

$$
\sum_{n=0}^{m} \sum_{j=1}^{k} p_{i j}^{n}(t) y_{j}^{(n)}(t)=f_{i}(t), i=1,2, \ldots \ldots, k
$$

This system can be written as follow

$$
\sum_{i=0}^{m} P_{i}(t) Y^{(i)}(t)=F(t)
$$

where the $p_{i j}^{n}(t)$ and $f_{i}(t)$ are well defined functions on the interval $[0, \infty)$ where the matrices $P_{i}(t), Y^{(i)}(t)$ and $F(t)$ on the form

$$
P_{i}(t)=\left(\begin{array}{cccc}
p_{11}^{i} & p_{12}^{i} & \cdots & p_{1 k}^{i} \\
p_{21}^{i} & p_{22}^{i} & \cdots & p_{2 k}^{i} \\
\vdots & \vdots & \vdots & \vdots \\
\vdots & \vdots & \vdots & \vdots \\
p_{k 1}^{i} & p_{k 2}^{i} & \cdots & p_{k k}^{i}
\end{array}\right), \quad Y^{(i)}(t)=\left(\begin{array}{c}
y_{1}^{(i)}(t) \\
y_{2}^{(i)}(t) \\
\vdots \\
\vdots \\
y_{k}^{(i)}(t)
\end{array}\right), \quad F(t)=\left(\begin{array}{c}
f_{1}(t) \\
f_{2}(t) \\
\vdots \\
\vdots \\
f_{k}(t)
\end{array}\right)
$$

We consider the above system under the mixed condition defined as

$$
\sum_{n=0}^{m-1} a_{i} Y^{(i)}(a)+b_{i} Y^{(i)}(b)+c_{i} Y^{(i)}(c)=\lambda, 0 \leqslant a \leqslant c \leqslant b<\infty,
$$

where $a_{i}, b_{i}, c_{i}$ and $\lambda$ are real valued vectors, and $a \rightarrow 0, b \rightarrow \infty$.

\section{The exponential Legendre functions}

In this section we list some properties of the EL functions.

\subsection{Orthogonality of EL functions}

The weight function $\omega(t)$ corresponding to the EL function, such that they are orthogonal in the interval $[0, \infty)$ is given by $2 e^{-t}$, with orthogonal condition

$$
\int_{0}^{\infty} E_{n}(t) E_{m}(t) \omega(t) d t=\frac{2}{2 n+1} \delta_{n m}
$$

where $\delta_{n m}$ is the Kronecker function.

\subsection{Function expansion in terms of EL functions}

A function $f(t)$ is well defined over the interval $[0, \infty)$ and can be expanded in terms of EL functions as

$$
f(t)=\sum_{n=0}^{\infty} a_{n} E_{n}(t)
$$

where

$$
a_{n}=\frac{2 n+1}{2} \int_{0}^{\infty} E_{n}(t) f(t) \omega(t) d t .
$$

If the summation in expression (3.1) is truncated to $N$ where $N<\infty$ it takes the following form

$$
f(t) \cong \sum_{n=0}^{N} a_{n} E_{n}(t)
$$

the $(k) t h$-order derivative of $f(t)$ can be written as

$$
f^{(k)}(t) \cong \sum_{n=0}^{N} a_{n}\left(E_{n}(t)\right)^{(k)}
$$

where $\left(E_{n}(t)\right)^{(0)}=E_{n}(t)$. 


\subsection{The operational matrix}

The new representation of EL functions is presented as follows.

If we use the expression $v(t)=1-2 e^{-t}$ in the EL functions, we can express it explicitly in terms of powers of $v(t)$ as

$$
E_{n}(t)=\sum_{k=0}^{[n 2]} q_{k}^{(n)} v^{n-2 k}(t)
$$

where

$$
q_{k}^{(n)}=(-1)^{k} \frac{1}{2^{n}}\left(\begin{array}{l}
n \\
k
\end{array}\right)\left(\begin{array}{c}
2 n-2 k \\
n
\end{array}\right), \quad n \geqslant 2 k
$$

and $[n 2]$ denotes the integer part of the value $\frac{n}{2}$.

From previous relation with simple modification we can define:

if $n$ is even number

$$
E_{2 l}(t)=\sum_{j=0}^{l}(-1)^{l-j} \frac{1}{2^{2 l}}\left(\begin{array}{c}
2 l \\
l-j
\end{array}\right)\left(\begin{array}{c}
2 l+2 j \\
2 l
\end{array}\right) v^{2 j}(t)
$$

if $n$ is odd number

$$
E_{2 l+1}(t)=\sum_{j=0}^{l}(-1)^{l-j} \frac{1}{2^{2 l+1}}\left(\begin{array}{c}
2 l+1 \\
l-j
\end{array}\right)\left(\begin{array}{c}
2 l+2 j+2 \\
2 l+1
\end{array}\right) v^{2 j+1}(t) .
$$

Form above relations we can deduce general matrix form of EL functions as

$$
E(t)=V(t) L^{T},
$$

where $E(t)$ and $V(t)$ are two matrices of the form:

$$
\begin{gathered}
E(t)=\left[\begin{array}{llll}
E_{0}(t) & E_{1}(t) & \cdots \cdots & E_{N}(t)
\end{array}\right], \\
V(t)=\left[\begin{array}{llll}
v^{0}(t) & v(t) & \cdots \cdots & v^{N}(t)
\end{array}\right]
\end{gathered}
$$

and $v^{0}(t)=1, \quad v^{1}(t)=1-2 e^{-t}, \quad v^{2}(t)=\left(1-2 e^{-t}\right)^{2}, \cdots \cdots, v^{N}(t)=\left(1-2 e^{-t}\right)^{N}$, and $L$ is a matrix given by

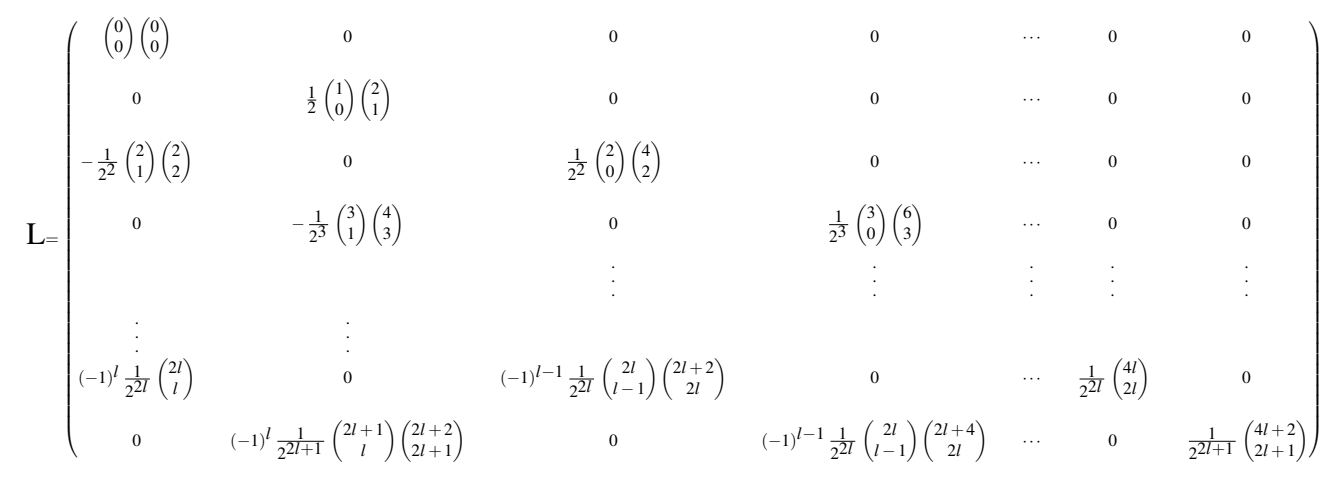

Now, from (3.3) we can obtain the $k^{t h}$ derivative of matrix $E(t)$ as:

$$
\begin{gathered}
E^{(0)}(t)=V(t) L^{T} \\
E^{(1)}(t)=V^{(1)}(t) L^{T} \\
E^{(2)}(t)=V^{(2)}(t) L^{T}
\end{gathered}
$$

then, by induction the $k^{t h}$-order derivative of the matrix $E(t)$ defined as:

$$
E^{(k)}(t)=V^{(k)}(t) L^{T}
$$

the equation (3.4) represents the new operational matrix of derivatives of the EL functions. 


\section{Fundamental matrix relations}

Let us define the collocation points, so that $0 \leqslant t_{s}<\infty$, as

$$
t_{s}=\frac{1+\cos \left(\frac{s \pi}{N}\right)}{1-\cos \left(\frac{s \pi}{N}\right)}
$$

and at the boundaries $(s=0, \quad s=N), t_{0} \rightarrow \infty, \quad t_{N} \rightarrow 0$, since the EL functions are convergent at both boundaries 0 and $\infty$, namely their values are \pm 1 , the appearance of infinity in the collocation points does not cause a loss or divergence in the method.

We assume that the solution $y_{i}(t)$ of (2.1) can be expressed in the form (3.2), which is a truncated Legendre series in terms of EL functions. Then $y_{i}(t)$ and its derivative $y_{i}^{(j)}(t)$ can be written in the matrix form as

$$
y_{i}(t)=E(t) A_{i}
$$

and

$$
y_{i}^{(j)}(t)=E^{(j)}(t) A_{i}, \quad i=1,2, \ldots \ldots, k, \quad j=0,1,2, \ldots \ldots, m
$$

where

$$
A_{i}=\left[a_{i 0}, a_{i 1} \ldots \ldots \ldots, a_{i N}\right]^{T}
$$

By substituting relation (3.4) into (4.1), we obtain

$$
y_{i}^{(j)}(t)=V^{(j)}(t) L^{T} A_{i}, \quad j=0,1,2, \ldots \ldots, m .
$$

So, the matrix $y^{(i)}(t)$ defined as a column matrix that is formed of $i^{t h}$ derivatives of unknown functions, can be expressed by

$$
y^{(i)}(t)=V^{(i)}(t) L^{T} A
$$

where $V^{(i)}(t), L^{T}$ are two size matrix $k \times k$,

$$
V^{(i)}(t)=\left(\begin{array}{cccc}
V^{(i)}(t) & 0 & \cdots & 0 \\
0 & V^{(i)}(t) & \cdots & 0 \\
\vdots & \vdots & \ddots & \vdots \\
0 & 0 & \cdots & V^{(i)}(t)
\end{array}\right), L^{T}=\left(\begin{array}{cccc}
L^{T} & 0 & \cdots & 0 \\
0 & L^{T} & \cdots & 0 \\
\vdots & \vdots & \ddots & \vdots \\
0 & 0 & \cdots & L^{T}
\end{array}\right), \quad A=\left(\begin{array}{c}
A_{0} \\
A_{1} \\
\vdots \\
A_{k}
\end{array}\right)_{k \times 1} .
$$

By putting the collocation points $t_{s}$ in (4.2), we have the system

$$
y^{(i)}\left(t_{s}\right)=V^{(i)}\left(t_{s}\right) L^{T} A,
$$

the system (4.3) can be written in the matrix form as

$$
Y^{(i)}=\widetilde{V}^{(i)} L^{T} A
$$

where

$$
\widetilde{V}^{(i)}=\left(\begin{array}{c}
V^{(i)}\left(t_{0}\right) \\
V^{(i)}\left(t_{1}\right) \\
\vdots \\
V^{(i)}\left(t_{N}\right)
\end{array}\right)
$$

then, the equation (2.2) becomes as follows

$$
\sum_{i=0}^{m} \widetilde{P}_{i} \widetilde{V}^{(i)} L^{T} A=F
$$

Next, we can obtain the corresponding matrices form for the conditions by using the relation (2.3), we have the fundamental matrix equation corresponding to the mixed conditions (2.3) as

$$
\sum_{i=0}^{m-1}\left[a_{i} V^{(i)}(a)+b_{i} V^{(i)}(b)+c_{i} V^{(i)}(c)\right] L^{T} A=\alpha
$$




\section{Collocation method}

The fundamental matrix (4.4) for equation (2.1) corresponding to system of $(N+1)$ algebraic equations for the $(N+1)$ unknown coefficients $a_{0,}, a_{1}, \ldots, a_{N}$. We can write equation (4.4) as

$$
H A=M
$$

So that $H=\left(h_{d, t}\right)=\sum_{i=0}^{m} \widetilde{P}_{i} \widetilde{V}^{(i)} L^{T}, \quad d, t=1,2, \ldots ., k(N+1)$.

We can obtain the matrix form for the mixed conditions by means of (4.5)

$$
R A=\left[\alpha_{i}\right]
$$

and

$$
R=\sum_{i=0}^{m-1}\left[a_{i} V^{(i)}(a)+b_{i} V^{(i)}(b)+c_{i} V^{(i)}(c)\right] L^{T}
$$

To obtain the solution of $\operatorname{Eq}(2.1)$ under the conditions (2.3) we replace the rows of matrices(5.2) by the last $m$ rows of the matrix (5.1) Then, we have the required augmented matrix as

$$
[\widetilde{R}, \widetilde{F}]=\left(\begin{array}{cccccc}
h_{1,1} & h_{1,2} & \cdots & h_{1, k(N+1)} & ; & f_{1}\left(t_{0}\right) \\
h_{2,1} & h_{2,2} & \cdots & h_{2, k(N+1)} & ; & f_{2}\left(t_{0}\right) \\
\vdots & \vdots & \vdots & \vdots & \vdots & \vdots \\
h_{k, 1} & h_{k, 2} & \cdots & h_{k, k(N+1)} & ; & f_{k}\left(t_{0}\right) \\
h_{k+1,1} & h_{k+1,2} & \cdots & h_{k+1, k(N+1)} & ; & f_{1}\left(t_{1}\right) \\
\vdots & \vdots & \vdots & \vdots & \vdots & \vdots \\
h_{k(N-m+1), 1} & h_{k(N-m+1), 2} & \cdots & h_{k(N-m+1), k(N+1)} & ; & f_{k}\left(t_{N-m}\right) \\
r_{1,1} & r_{1,2} & \cdots & r_{1, k(N+1)} & ; & \alpha_{1} \\
r_{2,1} & r_{2,2} & \cdots & r_{2, k(N+1)} & ; & \alpha_{2} \\
\vdots & \vdots & \vdots & \vdots & ; & \vdots \\
r_{m k, 1} & r_{m k, 2} & \cdots & r_{m k, k(N+1)} & ; & \alpha_{m k}
\end{array}\right)
$$

or the corresponding matrix equation

$$
\widetilde{R} A=\widetilde{F}
$$

then we can write

$$
A=(\widetilde{R})^{-1} \widetilde{F} .
$$

\section{Illustrative examples}

In this section, we demonstrate the effectiveness of the proposed Legendre exponential function method with numerical examples.

Example 6.1. Consider the system

$$
\left\{\begin{array}{c}
x^{\prime}-x-8 y=-12 e^{-2 t} \\
y^{\prime}-2 x-y=\frac{15}{4}-9 e^{-t}-\frac{9}{2} e^{-2 t} \quad, \quad 0 \leqslant t<\infty
\end{array}\right.
$$

with the conditions $x(0)=4, y(\infty)=\frac{1}{4}$, where the exact solution

$$
\left\{\begin{array}{c}
x(t)=-2+6 e^{-t} \\
y(t)=\frac{1}{4}-\frac{3}{2} e^{-t}+\frac{3}{2} e^{-2 t}
\end{array}\right.
$$

for this example we have

$$
k=2, \quad m=1, \quad f_{1}(t)=-12 e^{-2 t}, \quad f_{2}(t)=-\frac{9}{2} e^{-2 t}-9 e^{-t}+\frac{15}{4}
$$

and

$$
P_{0}=\left(\begin{array}{ll}
-1 & -8 \\
-2 & -1
\end{array}\right), \quad P_{1}=\left(\begin{array}{ll}
1 & 0 \\
0 & 1
\end{array}\right)
$$

Then, for $N=2$, the collocation points are $t_{0} \rightarrow \infty, t_{1} \rightarrow 1, t_{2} \rightarrow 0$, and the fundamental matrix is

$$
\left[\widetilde{P}_{0} \widetilde{V}^{(0)}+\widetilde{P}_{1} \widetilde{V}^{(1)}\right] L^{T} A=F
$$

where $\widetilde{P}_{0}, \widetilde{P}_{1}, \widetilde{V}^{(0)}$ and $\widetilde{V}^{(1)}$ are matrices of order $(6 \times 6)$ given as: 


$$
\begin{aligned}
& \widetilde{P}_{0}=\left(\begin{array}{cccccc}
-1 & -8 & 0 & 0 & 0 & 0 \\
-2 & -1 & 0 & 0 & 0 & 0 \\
0 & 0 & -1 & -8 & 0 & 0 \\
0 & 0 & -2 & -1 & 0 & 0 \\
0 & 0 & 0 & 0 & -1 & -8 \\
0 & 0 & 0 & 0 & -2 & -1
\end{array}\right), \widetilde{P}_{1}=\left(\begin{array}{cccccc}
1 & 0 & 0 & 0 & 0 & 0 \\
0 & 1 & 0 & 0 & 0 & 0 \\
0 & 0 & 1 & 0 & 0 & 0 \\
0 & 0 & 0 & 1 & 0 & 0 \\
0 & 0 & 0 & 0 & 1 & 0 \\
0 & 0 & 0 & 0 & 0 & 1
\end{array}\right), L^{T}=\left(\begin{array}{cccccc}
1 & 0 & -\frac{1}{2} & 0 & \frac{3}{8} & 0 \\
0 & 1 & 0 & -\frac{3}{2} & 0 & \frac{15}{8} \\
0 & 0 & \frac{3}{2} & 0 & -\frac{15}{4} & 0 \\
0 & 0 & 0 & \frac{5}{2} & 0 & -\frac{35}{4} \\
0 & 0 & 0 & 0 & \frac{35}{8} & 0 \\
0 & 0 & 0 & 0 & 0 & \frac{63}{8}
\end{array}\right) \\
& \widetilde{V}^{(0)}=\left(\begin{array}{cccccc}
1 & 1 & 1 & 0 & 0 & 0 \\
0 & 0 & 0 & 1 & 1 & 1 \\
1 & \left(1-2 e^{-1}\right) & \left(1-2 e^{-1}\right)^{2} & 0 & 0 & 0 \\
0 & 0 & 0 & 1 & \left(1-2 e^{-1}\right) & \left(1-2 e^{-1}\right)^{2} \\
1 & -1 & 1 & 0 & 0 & 0 \\
0 & 0 & 0 & 1 & -1 & 1
\end{array}\right) \\
& \widetilde{V}^{(1)}=\left(\begin{array}{cccccc}
0 & 0 & 0 & 0 & 0 & 0 \\
0 & 0 & 0 & 0 & 0 & 0 \\
0 & \left(2 e^{-1}\right) & \left(4 e^{-1}-8 e^{-2}\right) & 0 & 0 & 0 \\
0 & 0 & 0 & 0 & \left(2 e^{-1}\right) & \left(4 e^{-1}-8 e^{-2}\right) \\
0 & 2 & -4 & 0 & 0 & 0 \\
0 & 0 & 0 & 0 & 2 & -4
\end{array}\right)
\end{aligned}
$$

and $F=\left[\begin{array}{llllll}0 & \frac{15}{4} & -12 e^{-2} & -\frac{9}{2} e^{-2}-9 e^{-1}+\frac{15}{4} \quad-12 & -\frac{39}{4}\end{array}\right]$, and the augmented matrix for the conditions with $N=2$ is $\left[\begin{array}{l}1 \\ -\end{array}\right.$ $\left.\begin{array}{llllll}1 & 1 & 0 & 0 & 0 & ; 4\end{array}\right]$ for the first condition $x(0)=4$, and for the other condition $y(\infty)=\frac{1}{4}$, the augmented matrix is $\left[\begin{array}{llllllll}0 & 0 & 0 & 1 & 1 & 1 & ; & \frac{1}{4}\end{array}\right]$. After the augmented matrices of the system and condition are computed, we obtain the solution

$$
A=\left[\begin{array}{llllll}
1 & -3 & 0 & 0 & 0 & \frac{1}{4}
\end{array}\right]
$$

therefore, we find the approximate solutions as

$$
\left\{\begin{array}{c}
x(t)=E_{0}-3 E_{1}=1-3\left(1-2 e^{-t}\right)=1-3+6 e^{-t}=-2+6 e^{-t} \\
y(t)=0 E_{0}+0 E_{1}+\frac{1}{4} E_{2}=\frac{1}{4}\left[\frac{3}{2}\left(1-2 e^{-t}\right)^{2}-\frac{1}{2}\right]=\frac{1}{4}-\frac{3}{2} e^{-t}+\frac{3}{2} e^{-2 t}
\end{array}\right.
$$

Example 6.2. Consider the second order system of two equations

$$
\left\{\begin{array}{c}
y^{\prime \prime}+x^{\prime \prime}-x=90 e^{-3 t}-24 e^{-2 t}+\frac{23}{4} e^{-t}-3 \\
y^{\prime \prime}-2 x^{\prime}=90 e^{-3 t}-108 e^{-2 t}+\frac{119}{4} e^{-t} \quad, \quad 0 \leqslant t<\infty
\end{array}\right.
$$

with the conditions $x(0)=3, \quad y(\infty)=3, \quad x(\infty)=\frac{1}{2}, \quad y(0)=\frac{5}{4}$.

Where the exact solutions $x(t)=3-12 e^{-t}+12 e^{-2 t}$ and $y(t)=\frac{1}{2}+\frac{23}{4} e^{-t}-15 e^{-2 t}+10 e^{-3 t}$.

In this system, we have

$$
k=2, \quad m=2, \quad f_{1}(t)=90 e^{-3 t}-24 e^{-2 t}+\frac{23}{4} e^{-t}-3, \quad f_{2}(t)=90 e^{-3 t}-108 e^{-2 t}+\frac{119}{4} e^{-t}
$$

and

$$
P_{0}=\left(\begin{array}{cc}
-1 & 0 \\
0 & 0
\end{array}\right), \quad P_{1}=\left(\begin{array}{ll}
0 & 0 \\
2 & 0
\end{array}\right), \quad P_{2}=\left(\begin{array}{ll}
1 & 1 \\
0 & 1
\end{array}\right)
$$

Then, for $N=4$, the collocation points are $t_{0} \rightarrow \infty, t_{1} \rightarrow 3+2 \sqrt{2}, t_{2} \rightarrow 1, t_{3} \rightarrow 3-2 \sqrt{2}, t_{4} \rightarrow 0$, and the fundamental matrix is

$$
\left[\widetilde{P}_{0} \widetilde{V}^{(0)}+\widetilde{P}_{1} \widetilde{V}^{(1)}+\widetilde{P}_{2} \widetilde{V}^{(2)}\right] L^{T} A=F
$$

where $\widetilde{P}_{0}, \widetilde{P}_{1}, \widetilde{P}_{2}, \widetilde{V}^{(0)}, \widetilde{V}^{(1)}$ and $\widetilde{V}^{(2)}$ are matrices of order $(10 \times 10)$

$$
\widetilde{P}_{0}=\left(\begin{array}{cccccccccc}
-1 & 0 & 0 & 0 & 0 & 0 & 0 & 0 & 0 & 0 \\
0 & 0 & 0 & 0 & 0 & 0 & 0 & 0 & 0 & 0 \\
0 & 0 & -1 & 0 & 0 & 0 & 0 & 0 & 0 & 0 \\
0 & 0 & 0 & 0 & 0 & 0 & 0 & 0 & 0 & 0 \\
0 & 0 & 0 & 0 & -1 & 0 & 0 & 0 & 0 & 0 \\
0 & 0 & 0 & 0 & 0 & 0 & 0 & 0 & 0 & 0 \\
0 & 0 & 0 & 0 & 0 & 0 & -1 & 0 & 0 & 0 \\
0 & 0 & 0 & 0 & 0 & 0 & 0 & 0 & 0 & 0 \\
0 & 0 & 0 & 0 & 0 & 0 & 0 & 0 & -1 & 0 \\
0 & 0 & 0 & 0 & 0 & 0 & 0 & 0 & 0 & 0
\end{array}\right), \widetilde{P}_{1}=\left(\begin{array}{cccccccccc}
0 & 0 & 0 & 0 & 0 & 0 & 0 & 0 & 0 & 0 \\
2 & 0 & 0 & 0 & 0 & 0 & 0 & 0 & 0 & 0 \\
0 & 0 & 0 & 0 & 0 & 0 & 0 & 0 & 0 & 0 \\
0 & 0 & 2 & 0 & 0 & 0 & 0 & 0 & 0 & 0 \\
0 & 0 & 0 & 0 & 0 & 0 & 0 & 0 & 0 & 0 \\
0 & 0 & 0 & 0 & 2 & 0 & 0 & 0 & 0 & 0 \\
0 & 0 & 0 & 0 & 0 & 0 & 0 & 0 & 0 & 0 \\
0 & 0 & 0 & 0 & 0 & 0 & 2 & 0 & 0 & 0 \\
0 & 0 & 0 & 0 & 0 & 0 & 0 & 0 & 0 & 0 \\
0 & 0 & 0 & 0 & 0 & 0 & 0 & 0 & 2 & 0
\end{array}\right),
$$




$$
\begin{aligned}
& \widetilde{P_{2}}=\left(\begin{array}{llllllllll}
1 & 1 & 0 & 0 & 0 & 0 & 0 & 0 & 0 & 0 \\
0 & 1 & 0 & 0 & 0 & 0 & 0 & 0 & 0 & 0 \\
0 & 0 & 1 & 1 & 0 & 0 & 0 & 0 & 0 & 0 \\
0 & 0 & 0 & 1 & 0 & 0 & 0 & 0 & 0 & 0 \\
0 & 0 & 0 & 0 & 1 & 1 & 0 & 0 & 0 & 0 \\
0 & 0 & 0 & 0 & 0 & 1 & 0 & 0 & 0 & 0 \\
0 & 0 & 0 & 0 & 0 & 0 & 1 & 1 & 0 & 0 \\
0 & 0 & 0 & 0 & 0 & 0 & 0 & 1 & 0 & 0 \\
0 & 0 & 0 & 0 & 0 & 0 & 0 & 0 & 1 & 1 \\
0 & 0 & 0 & 0 & 0 & 0 & 0 & 0 & 0 & 1
\end{array}\right), \\
& L^{T}=\left(\begin{array}{cccccccccc}
1 & 0 & -\frac{1}{2} & 0 & \frac{3}{8} & 0 & -\frac{15}{6} & 0 & \frac{35}{128} & 0 \\
0 & 1 & 0 & -\frac{3}{2} & 0 & \frac{15}{8} & 0 & -\frac{35}{16} & 0 & \frac{315}{128} \\
0 & 0 & \frac{3}{2} & 0 & -\frac{15}{4} & 0 & \frac{105}{16} & 0 & -\frac{1260}{128} & 0 \\
0 & 0 & 0 & \frac{5}{2} & 0 & -\frac{35}{4} & 0 & \frac{315}{16} & 0 & -\frac{4620}{128} \\
0 & 0 & 0 & 0 & \frac{35}{8} & 0 & -\frac{35}{16} & 0 & \frac{6930}{128} & 0 \\
0 & 0 & 0 & 0 & 0 & \frac{63}{8} & 0 & -\frac{693}{16} & 0 & \frac{1801}{128} \\
0 & 0 & 0 & 0 & 0 & 0 & \frac{231}{16} & 0 & -\frac{12012}{128} & 0 \\
0 & 0 & 0 & 0 & 0 & 0 & 0 & \frac{429}{16} & 0 & -\frac{25740}{128} \\
0 & 0 & 0 & 0 & 0 & 0 & 0 & 0 & \frac{6435}{128} & 0 \\
0 & 0 & 0 & 0 & 0 & 0 & 0 & 0 & 0 & \frac{12155}{128}
\end{array}\right)
\end{aligned}
$$

and the augmented matrix for the conditions with $N=4$ is $\left[\begin{array}{llllllllll}1 & -1 & 1 & -1 & 1 & 0 & 0 & 0 & 0 & 0 ;\end{array}\right]$ for the first condition $x(0)=$ 3 , and $y(\infty)=3$, the augmented matrix is $\left[\begin{array}{lllllllllll}0 & 0 & 0 & 0 & 0 & 1 & 1 & 1 & 1 & 1 ;\end{array}\right]$, and for the other condition $x(\infty)=\frac{1}{2}$ we have $\left[\begin{array}{lllllllllll}1 & 1 & 1 & 1 & 1 & 0 & 0 & 0 & 0 & 0 ; & \frac{1}{2}\end{array}\right]$ and $y(0)=\frac{5}{4}$ we have the augmented matrix $\left[\begin{array}{ccccccccccc}0 & 0 & 0 & 0 & 0 & 1 & -1 & 1 & -1 & 1 ; & 5\end{array}\right]$. After the augmented matrices of the system and condition are computed, we obtain the solution

$$
A=\left[\begin{array}{llllllllll}
1 & 0 & 2 & 0 & 0 & 0 & 1 & 0 & -\frac{1}{2} & 0
\end{array}\right] .
$$

Finally, we find the approximate solutions as

$$
\left\{\begin{array}{c}
x(t)=E_{0}+2 E_{2}=1+2\left[\frac{3}{2}\left(1-2 e^{-t}\right)^{2}-\frac{1}{2}\right]=3\left(1-2 e^{-t}\right)^{2} \\
y(t)=E_{1}-\frac{1}{2} E_{3}=\frac{7}{4}-\frac{7}{4} e^{-t}-\frac{5}{4}\left(1-2 e^{-t}\right)^{3}
\end{array}\right.
$$

\section{Conclusion}

Systems of high order linear differential equations are generally difficult to solve analytically under mixture conditions. In many cases, obtaining approximate solutions is necessary especially if the problem is defined in semi-infinite domain. For this reason, the Legendre exponential collocation method is proposed to obtain an approximate solution of high order linear differential equation. The new definition of EL functions is studied and introduced to solve the system of high order linear ordinary differential equations with constant coefficients. In addition, an interesting feature of this method is to find the exact solutions if the system has an exact solution that is a polynomial exponential function. Examples of problems are used to demonstrate the applicability and effectiveness of the proposed technique.

\section{References}

[1] M. Elarbi Benattia, K. Belghaba. Application of the Galerkin Method with Chebyshev Polynomials for Solving the Integral Equation.Journal of Computer Science \& Computational Mathematics, DOI: 10.20967/jcscm.2017.03.008.

[2] M. Elarbi Benattia, K. Belghaba. Numerical Solution for Solving Fractional Differential Equations using Shifted Chebyshev Wavelet. General Letters in Mathematics Vol. 3, No. 2, Oct 2017, pp.101-110.

[3] Mohamed A. Ramadana, Kamal R. Raslanb, Talaat S. El Danafa, Mohamed A. Abd El Salamb. Solving systems of high-order ordinary differential equations with variable coefficients by exponential Chebyshev collocation method. Journal of Modern Methods in Numerical Mathematics 8:1-2 (2017), 40-51.

[4] Mehdi Dehghan, Mohammad Shakourifar, and Asgar Hamidi, The solution of linear and nonlinear systems of volterra functional equations using adomian-pade technique, Chaos, Solutions \& Fractals 39 (2009), no. 5, 2509-2521.

[5] Elcin Gokmen and Mehmet Sezer, Taylor collocation method for systems of high-order linear differential-difference equations with variable coefficients, Ain Shams Engineering Journal 4 (2013), no. 1, 117-125.

[6] F. Baharifard - Saeed Kazem - K. Parand. Rational and Exponential Legendre Tau Method on Steady Flow of a Third Grade Fluid in a Porous Half Space, Int. J. Appl. Comput. Math (2016) 2:679-698.

[7] Hossein Jafari and Varsha Daftardar-Gejji, Revised adomian decomposition method for solving systems of ordinary and fractional differential equations, Applied Mathematics and Computation 181 (2006), no. 1, 598-608.

[8] IH Abdel-Halim Hassan, Application to differential transformation method for solving systems of differential equations, Applied Mathematical Modeling 32 (2008), no. 12, 2552-2559.

[9] Nebiye Korkmaz and Mehmet Sezer, An approach to numerical solutions of system of high-order linear differential-difference equations with variable coefficients and error estimation based on residual function, New Trends in Mathematical Sciences 2 (2014), no. 3, 220-233. 
[10] Mehdi Tatari and Mehdi Dehghan, Improvement of hes variational iteration method for solving systems of differential equations, Computers \& Mathematics with Applications 58 (2009), no. 11, 2160-2166.

[11] Montri Thongmoon and Sasitorn Pusjuso, The numerical solutions of differential transform method and the Laplace transform method for a system of differential equations, Nonlinear Analysis: Hybrid Systems 4 (2010), 425-431.

[12] Salih Yalc snbas, Nesrin Ozsoy, and Mehmet Sezer, Approximate solution of higher order linear dierential equations by means of a new rational chebyshev collocation method, Mathematical \& Computational Applications 15 (2010), no. 1, 45-56. 